\title{
Transition to turbulence in shock-driven mixing: a Mach number study
}

\author{
M. Lombardini $\dagger$, D. I. Pullin and D. I. Meiron \\ Graduate Aeronautical Laboratories, California Institute of Technology, Pasadena, CA 91125, USA
}

(Received 8 March 2011; revised 12 July 2011; accepted 25 September 2011; first published online 21 November 2011)

Large-eddy simulations of single-shock-driven mixing suggest that, for sufficiently high incident Mach numbers, a two-gas mixing layer ultimately evolves to a late-time, fully developed turbulent flow, with Kolmogorov-like inertial subrange following a $-5 / 3$ power law. After estimating the kinetic energy injected into the diffuse density layer during the initial shock-interface interaction, we propose a semi-empirical characterization of fully developed turbulence in such flows, based on scale separation, as a function of the initial parameter space, as $\left(\eta_{0^{+}} \Delta u / v\right)\left(\eta_{0^{+}} / L_{\rho}\right) A^{+} / \sqrt{1-A^{+^{2}}} \gtrsim$ $1.53 \times 10^{4} / \mathscr{C}^{2}$, which corresponds to late-time Taylor-scale Reynolds numbers $\gtrsim 250$. In this expression, $\eta_{0^{+}}$represents the post-shock perturbation amplitude, $\Delta u$ the change in interface velocity induced by the shock refraction, $v$ the characteristic kinematic viscosity of the mixture, $L_{\rho}$ the inner diffuse thickness of the initial density profile, $A^{+}$the post-shock Atwood ratio, and $\mathscr{C}\left(A^{+}, \eta_{0^{+}} / \lambda_{0}\right) \approx 0.3$ for the gas combination and post-shock perturbation amplitude considered. The initially perturbed interface separating air and $\mathrm{SF}_{6}$ (pre-shock Atwood ratio $A \approx 0.67$ ) was impacted in a heavy-light configuration by a shock wave of Mach number $M_{I}=1.05,1.25$, $1.56,3.0$ or 5.0 , for which $\eta_{0^{+}}$is fixed at about $25 \%$ of the dominant wavelength $\lambda_{0}$ of an initial, Gaussian perturbation spectrum. Only partial isotropization of the flow (in the sense of turbulent kinetic energy and dissipation) is observed during the late-time evolution of the mixing zone. For all Mach numbers considered, the late-time flow resembles homogeneous decaying turbulence of Batchelor type, with a turbulent kinetic energy decay exponent $n \approx 1.4$ and large-scale $(k \rightarrow 0)$ energy spectrum $\sim k^{4}$, and a molecular mixing fraction parameter, $\Theta \approx 0.85$. An appropriate time scale characterizing the Taylor-scale Reynolds number decay, as well as the evolution of mixing parameters such as $\Theta$ and the effective Atwood ratio $A_{e}$, seem to indicate the existence of low- and high-Mach-number regimes.

Key words: shock waves, transition to turbulence, turbulent mixing

\section{Introduction}

Consider a planar incident shock of Mach number $M_{I}$ that encounters a perturbed, planar density interface. Owing to shock impact, the interface is impulsively accelerated at a velocity $\Delta u$, which depends on the gas combination and $M_{I}$. During the initial shock refraction phase, the interface is compressed and baroclinic vorticity 
is generated as a result of the misalignment between the pressure gradient across the shock and the local density gradient at the deformed interface. In the early stages of the growth, the peaks and troughs of the interface are expected to grow linearly in time, as predicted by Richtmyer (1960). When the perturbation amplitude approaches its characteristic wavelength, the growth becomes nonlinear, with peaks and troughs evolving asymmetrically, as spikes (heavy fluid penetrating light fluid) and bubbles (light fluid penetrating heavy fluid). Secondary Kelvin-Helmholtz instabilities appear along the fingering structures of the distorted interface, and mixing may eventually occur at small scales.

In the dynamics of mixing, pockets of one fluid species are entrained into the other one on either the light or heavy side of the mixing region as a consequence of large-scale motions. The induced kinematic stirring stretches the two fluids and increases the surface of contact between the mixing species, generating scales below the largest eddy sizes. Ever-decreasing scales are subsequently produced, the smallest ones being eventually affected by viscosity and molecular diffusivity. The mixing process is considered to have transitioned to fully developed turbulence when a wide enough spectrum of scales has been generated, increasing the interfacial area between the two fluids and thereby intensifying mixing. While this mixing transition reveals itself through changes in mixing properties (e.g. scalar field), it can also be quantified by other measures, as will be discussed below.

Fully developed turbulent flows generally require a Reynolds number $R e$ to be defined that is high enough. Assessing a large number of experiments (jets, shear layers, Couette-Taylor flows, etc.), Dimotakis (2000), following Konrad (1976), proposed that a mixing transition occurs in stationary flows when the Taylor-scale Reynolds number, $R e_{\lambda}=u^{\prime} \lambda_{T} / v \sim \sqrt{R e} \gtrsim 100-140$, where $u^{\prime}$ is the root-mean-square (r.m.s.) velocity fluctuation level, $\lambda_{T}$ the Taylor microscale and $v$ the characteristic kinematic viscosity of the mixture. In that case, there is three orders of magnitude separation between the large-eddy, driving scales and the smallest, dissipative scales, allowing an inertial range that is decoupled from both. A similar scale-separation requirement was drawn by Pullin, Buntine \& Saffman (1994) who modelled turbulence as an ensemble of stretched-spiral vortices (Lundgren 1982). Relating the size of the spiral structures to the Taylor microscale and the integral scale $\ell$, scale separation between $\ell$ and the maximum stretched length of the vortices was found for $R e_{\lambda} \gtrsim 100$.

However, for unsteady flows such as shock-accelerated mixing, transition cannot occur until an inertial subrange has had time to develop, even for $R e_{\lambda}$ sufficiently large that the inertial subrange can theoretically exist. In other words, $R e$ or $R e_{\lambda}$ large enough is a necessary but not sufficient condition as these large values must be sustained for a finite period of time for a turbulent flow to develop. Zhou, Robey \& Buckingham (2003) translated the criterion of Dimotakis to a time constraint for nonstationary, accelerated flows, identifying $R e$ with the time-dependent Reynolds number $\delta \dot{\delta} / v$ based on the evolution of the mixing-layer width $\delta(t)$ and its growth rate $\dot{\delta}(t)$ at time $t$, and assuming that $\delta \sim t^{\theta}$. Within this theory, an inertial range can only appear if $\theta>1 / 2$. In addition, while this analysis could probably predict localized transition to molecular mixing, induced for example by secondary instabilities within the rollups of single-mode spikes, it does not account for the generation of larger scales by nonlinear coupling between modes of an initially broader perturbation spectrum, and subsequent effects on the inertial subrange development at the low-wavenumber end of the spectrum.

The mixing transition in single-shock-accelerated flows has been mostly investigated in gas curtain experiments. Vorobieff, Rightley \& Benjamin (1998) showed that for 
multimode perturbations the intensity structure function reached a $-5 / 3$ power law. Transition to turbulence was also studied using fractal dimension theory (Vorobieff, Rightley \& Benjamin 1999) and histogram analysis (Rightley et al. 1999; Prestridge et al. 2000). In more recent experiments on a heavy-gas $\left(\mathrm{SF}_{6}\right)$ curtain at $M_{I}=1.2$, Balakumar et al. (2008) performed particle-image velocimetry (PIV) and planar laserinduced fluorescence (PLIF) measurements, leading to histograms of the fluctuating axial and transverse velocities that were compared to the mean flow velocity as an estimate of the turbulent intensity. A wider range of Mach numbers, $M_{I}=1.2$, 1.5 and 2.0, was investigated by Orlicz et al. (2009) using the same experimental apparatus: the instantaneous mixing rate was estimated from quantitative concentration fields extracted from PLIF images (Tomkins et al. 2008), and provided evidence of a stronger mixing as $M_{I}$ is increased.

Among the main relevant studies of single-shock-induced mixing at a single contact surface, Jacobs \& Krivets (2005) presented experiments on a planar, membraneless air- $\mathrm{SF}_{6}$ interface impacted by relatively weak Mach numbers, $M_{I}=1.1,1.2$ and 1.3 in a light-to-heavy configuration. PLIF provided detailed images of the disintegration of initial, large vortical structures into smaller, disordered scales at which molecular mixing occurs. In similar membraneless experiments, Motl et al. (2009) computed a vortex Reynolds number based on the circulation related to the vorticity deposition phase (Glezer 1988; Samtaney \& Zabusky 1994), and identified it with the outerscale Reynolds number $R e$ used by Dimotakis (2000) as an indication of the mixing transition.

For Richtmyer-Meshkov instability with reshock, as in shock tube experiments with an end wall (Vetter \& Sturtevant 1995; Collins \& Jacobs 2002; Leinov et al. 2009), the second shock interaction deposits additional baroclinic vorticity into an already growing mixing zone, distributing the energy across a wider spectrum of scales (Hill, Pantano \& Pullin 2006). In such conditions, transition to a fully developed turbulent flow is clearly observed immediately after the reshock, or possible secondary reverberations following the reshock as described for example in Lombardini et al. (2011). Single-shock interactions, however, do not always exhibit clear signs of transition, specially for low levels of initial baroclinic energy deposition (e.g. for low Atwood ratio $A$ or $M_{I}$ ). The following questions can therefore be posed: $(a)$ for what sub-domain of the initial parameter space has transition occurred? If transition is observed, (b) at what time has transition taken place, and $(c)$ what are the characteristics of the late-time turbulent state that the flow transitioned to?

We present a computational investigation based on large-eddy simulation (LES) of single-shock impact on a single interface at five distinct Mach numbers. After describing the geometry, initial conditions and computational strategy in $\S 2$, we propose in $\S 3$ a predictive tool for assessing the existence of fully developed turbulent mixing induced by a shock wave. Some characteristics of the turbulence are exposed in $\S 4$ (decay of turbulent kinetic energy) and $\S 5$ (kinetic energy power spectra). Finally, various statistics on the mixing accompanying the transition are presented in $\S 6$.

\section{Description of the problem and computational approach}

\subsection{Geometry}

The flow is computed in the frame of a density interface accelerated in a heavy-light configuration by a shock wave propagating in the direction of increasing $x$. To facilitate interpretation of statistical results and to exclude boundary layer issues from 
the simulations, periodic boundary conditions are used in the $(y, z)$-plane transverse to the $x$-direction. With homogeneity in the $y$ - and $z$-directions, ensemble averages are equivalent to integrals in the transverse directions, and for any variable $Q(x, y, z ; t)$ we denote by $\langle Q\rangle(x, t)$ its plane average, and by $Q^{\prime}(x, y, z ; t)$ the local fluctuation from this average. Similarly to the shock tube experiments of Vetter \& Sturtevant (1995), we set $L_{y}=L_{z}=L=0.27 \mathrm{~m}$ as the transverse widths of the domain. The computational domain is discretized in cubic cells of size $\Delta=L / N \approx 0.0021 \mathrm{~m}$, where the resolution in the transverse directions $y$ and $z$ is $N=128$. In the $x$-direction, $L_{x}=0.4 \mathrm{~m}$ allows the interface to grow sufficiently before reaching both ends of the domain. There, time-dependent boundary conditions are tailored such that the transmitted shock and reflected expansion exit without spurious waves reflecting back into the computational domain.

\subsection{Initial interfacial perturbation}

We define by $x-\xi(y, z)=0$ the surface where light and heavy fluids have equal mass fractions. The initial heavy-fluid mass fraction $\psi$ takes the form of a hyperbolic tangent profile centred at $\xi(y, z)$ and with a characteristic thickness $L_{\rho}=0.01 \mathrm{~m}$ (i.e. $L_{\rho}$ is the characteristic length scale of the unperturbed density profile):

$$
\psi(x, y, z ; 0)=\frac{1}{2}-\frac{1}{2} \tanh \left[\frac{x-\xi(y, z)}{L_{\rho}}\right],
$$

where $\xi(y, z)$ is a field of perturbations represented by a wave packet of modes with random phase and whose power spectrum $E_{\xi}\left(k_{y}, k_{z}\right)$, with $k_{y}$ and $k_{z}$ the wavenumbers in both transverse directions, has the following annular Gaussian profile centred at the wavenumber $k_{0}$ and with variance $\sigma_{0}$ :

$$
E_{\xi}\left(k_{r}\right)=\frac{1}{32 \pi^{3} \sqrt{2 \pi}} \frac{\eta_{0}^{2} L^{2}}{k_{0} \sigma_{0}} \exp \left[-\frac{\left(k_{r}-k_{0}\right)^{2}}{2 \sigma_{0}^{2}}\right],
$$

where $k_{r}=\sqrt{k_{y}^{2}+k_{z}^{2}}$ defines the radial wavenumber and $\eta_{0}$ represents the perturbation amplitude. The dominant wavenumber is set to $k_{0}=2 \pi / \lambda_{0}$, where $\lambda_{0}=0.027 \mathrm{~m}$, that is $(L / 2 \pi) k_{0}=10$ periods per shock tube width and $N / 10$ points per wavelength. We choose $\sigma_{0}=k_{0} / 5$ such that the extent of the Gaussian in the radial wavenumber space (roughly $k_{0}-3 \sigma_{0}<k_{r}<k_{0}-3 \sigma_{0}$ ) is contained within the resolved wavenumber space $[2 \pi / L, \pi / \Delta]$, where $(L / 2 \pi) k_{\max }=L /(2 \Delta)=64$ defines to the Nyquist wavenumber. The Gaussian spectrum is shown in figure 1, and the corresponding initial perturbations are depicted in figure 2 .

The interface displacement variance $\int_{0}^{\infty} 2 \pi k_{r} E_{\xi}\left(k_{r}\right) \mathrm{d} k_{r}$ for such a multimode perturbation is set to be equal to the displacement variance of the two-dimensional, single-mode perturbation with wavenumber $k_{0}$ in the $y$ - and $z$-directions and maximum amplitude $\eta_{0}$,

$$
\xi(y, z)=\frac{\eta_{0}}{2}\left[\cos \left(k_{0} y\right)+\cos \left(k_{0} z\right)\right]
$$

In other words, the r.m.s. of the present multi-mode perturbation and its single-mode equivalent are both equal and given by:

$$
\sqrt{\frac{1}{L^{2}} \int_{-L / 2}^{L / 2} \int_{-L / 2}^{L / 2} \xi^{2} \mathrm{~d} y \mathrm{~d} z}=\frac{\eta_{0}}{2} .
$$




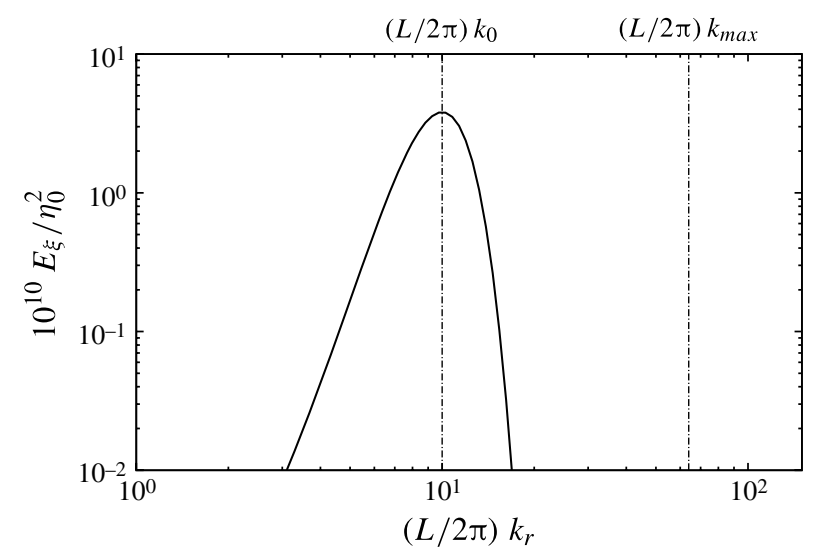

FIGURE 1. Spectrum of initial interfacial perturbations.

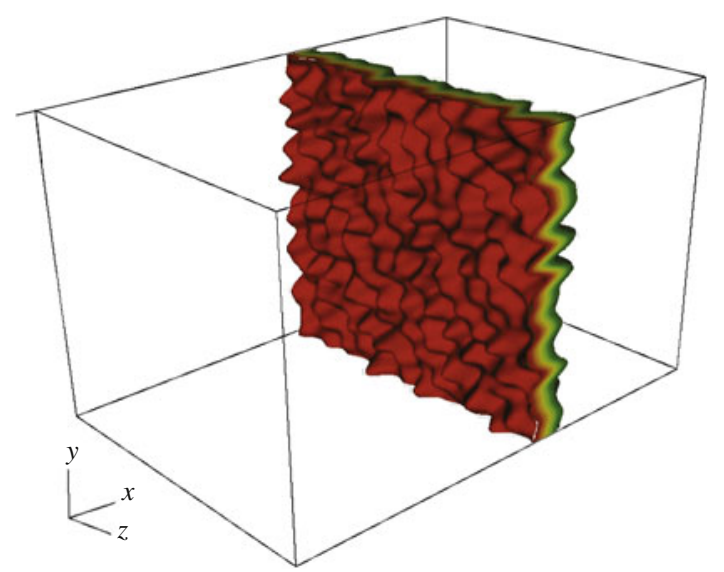

FIGURE 2. Initial isosurfaces of the heavy-fluid mass fraction $\psi$, with $0.01<\psi<0.99$ displayed.

Owing to the initial shock refraction phase, the initial perturbation amplitude $\eta_{0}$ is decreased to a minimal value $\eta_{0^{+}}$immediately after the shock impact and before the perturbation starts growing. A typical estimate of the early-time perturbation growth is given by the impulsive model of Richtmyer (1960) that predicts a growth rate of $k_{0} \eta_{0^{+}} A^{+} \Delta u$, which contains the dependence on the post-shock amplitude-towavelength ratio, post-shock Atwood ratio and Mach number. Considering that $A^{+}$is weakly varying with $M_{I}$, the effect of $M_{I}$ is isolated by setting $\eta_{0}$ to a value such that $\eta_{0^{+}}$is fixed for any of the Mach numbers considered. We choose $\eta_{0^{+}} / \lambda_{0} \approx 0.25$, large enough to precipitate the nonlinear development of the perturbation. Figure 3 recapitulates the main wavelengths involved in the initial problem, while table 1 lists the different values of $M_{I}$ tested.

\subsection{Large-eddy equations for two-gas mixing}

As observed in shock-driven mixing experiments and computations, the shock-interface interaction can produce a large dynamical range of scales that requires, with current computational resources, the use of LES. In this approach, the smallest 


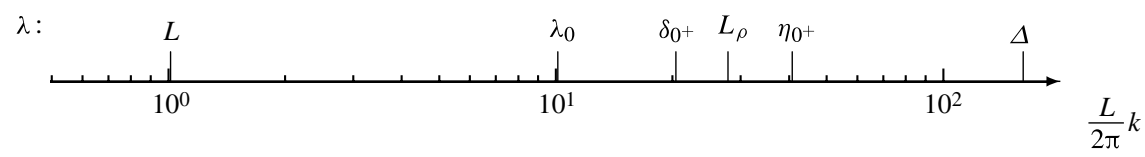

FIGURE 3. Important lengths $\lambda$ of the initial problem, and associated wavenumbers $k=$ $2 \pi / \lambda$ : transverse length of the domain $L$, dominant wavelength $\lambda_{0}$ of the perturbation, postshock width of the mixing layer $\delta_{0^{+}}$, intrinsic thickness of the initially diffuse unperturbed density profile $L_{\rho}$, post-shock amplitude $\eta_{0^{+}}$of the perturbation, and grid size $\Delta$ (also equal to the LES subgrid cutoff scale).

$\begin{array}{lccccc}M_{I} & 1.05 & 1.25 & 1.56 & 3.0 & 5.0 \\ & & & & & \\ \eta_{0^{+}} / \lambda_{0} & 0.25 & 0.25 & 0.25 & 0.25 & 0.25 \\ A^{+} & 0.67 & 0.69 & 0.70 & 0.73 & 0.75 \\ \Delta u\left(\mathrm{~m} \mathrm{~s}^{-1}\right) & 17 & 76 & 141 & 438 & 768\end{array}$

TABLE 1. Table of runs and important parameters for an $\mathrm{SF}_{6}$-air heavy-light shock interaction. The temperature, pressure and Atwood ratio for the pre-shock configuration are $T_{0}=286 \mathrm{~K}, p_{0}=23 \mathrm{kPa}$ and $A=0.67$ respectively.

scales of motion are conceptually removed by applying an homogeneous low-pass filter to the Navier-Stokes equations with spatial width usually corresponding to the finest grid resolution employed, $\Delta$. The resulting equations govern the transport of the filtered density $\bar{\rho}$, momentum $\bar{\rho} \widetilde{u}_{i}$, total energy $\bar{E}$ of the mixture, and the density $\bar{\rho} \widetilde{\psi}$ of the heavy fluid that describe the motion of the large, resolved scales well (Lombardini et al. 2011):

$$
\begin{gathered}
\frac{\partial \bar{\rho}}{\partial t}+\frac{\partial \bar{\rho} \widetilde{u}_{j}}{\partial x_{j}}=0, \\
\frac{\partial \widetilde{\rho}_{i}}{\partial t}+\frac{\partial\left(\bar{\rho} \widetilde{u}_{i} \widetilde{u}_{j}+\bar{p} \delta_{i j}\right)}{\partial x_{j}}-\frac{\partial \check{\sigma}_{i j}}{\partial x_{j}}=-\frac{\partial \tau_{i j}}{\partial x_{j}}, \\
\frac{\partial \bar{E}}{\partial t}+\frac{\partial(\bar{E}+\bar{p}) \widetilde{u}_{j}}{\partial x_{j}}-\frac{\partial}{\partial x_{j}}\left(\check{\sigma}_{j i} \widetilde{u}_{i}\right)=-\frac{\partial}{\partial x_{j}}\left(q_{j}^{T}-q_{j}^{T \partial \psi}\right) \\
\frac{\partial \bar{\rho} \widetilde{\psi}}{\partial t}+\frac{\partial \bar{\rho} \widetilde{\psi} \widetilde{u}_{j}}{\partial x_{j}}+\frac{\partial \check{J}_{j}}{\partial x_{j}}=-\frac{\partial q_{j}^{\psi}}{\partial x_{j}},
\end{gathered}
$$

where the filtered Newtonian stress tensor $\check{\sigma}_{i j}$, conductive heat flux $\check{q}_{c_{j}}$, interdiffusional enthalpy flux $\breve{q}_{d_{j}}$ and diffusive mass flux $\breve{J}_{j}$ are given by

$$
\begin{gathered}
\check{\sigma}_{i j}=\bar{\mu}\left[\left(\frac{\partial \widetilde{u}_{i}}{\partial x_{j}}+\frac{\partial \widetilde{u}_{j}}{\partial x_{i}}\right)-\frac{2}{3} \frac{\partial \widetilde{u}_{k}}{\partial x_{k}} \delta_{i j}\right], \quad \bar{\mu} \equiv \mu(\widetilde{\psi}, \widetilde{T}), \\
\check{q}_{c_{j}}=-\bar{\kappa} \frac{\partial \widetilde{T}}{\partial x_{j}}, \quad \bar{\kappa} \equiv \kappa(\widetilde{\psi}, \widetilde{T}), \\
\breve{q}_{d_{j}}=-\bar{\rho} \widetilde{D} \widetilde{T}\left(c_{p_{h}}-c_{p_{l}}\right) \frac{\partial \widetilde{\psi}}{\partial x_{j}}, \quad \widetilde{D} \equiv D(\widetilde{T}),
\end{gathered}
$$


Property

Molecular mass $m_{\alpha}\left(\mathrm{kg} \mathrm{kmol}^{-1}\right)$

Ratio of specific heats $\gamma_{\alpha}$

Density $\rho_{\alpha}\left(\mathrm{kg} \mathrm{m}^{-3}\right)$

Kinematic viscosity $v_{\alpha}\left(10^{-6} \mathrm{~m}^{2} \mathrm{~s}^{-1}\right)$

Prandtl number

Diffusion coefficient in air $D_{\alpha}\left(10^{-6} \mathrm{~m}^{2} \mathrm{~s}^{-1}\right)$
Air $\quad \mathrm{SF}_{6}$

$\begin{array}{ll}28.83 & 146.07\end{array}$

$\begin{array}{ll}1.40 & 1.09\end{array}$

$1.18 \quad 5.97$

$15.7 \quad 2.47$

$0.71 \quad 0.90$

$20.4 \quad 9.7$

TABLE 2. Gas properties of air and $\mathrm{SF}_{6}$ at $25^{\circ} \mathrm{C}$ and $1 \mathrm{~atm}$.

$$
\check{J}_{j}=-\bar{\rho} \widetilde{D} \frac{\partial \widetilde{\psi}}{\partial x_{j}},
$$

and the filtered pressure and temperature fields by

$$
\begin{gathered}
\bar{p}=(\widetilde{\gamma}-1)\left\{\bar{E}-\frac{1}{2} \bar{\rho} \widetilde{u}_{i} \widetilde{u}_{i}-\frac{1}{2} \tau_{i i}+\left[\widetilde{c}_{p} \widetilde{m}\left(\frac{1}{m_{h}}-\frac{1}{m_{l}}\right)-\left(c_{p_{h}}-c_{p_{l}}\right)\right] \phi^{T \psi}\right\}, \\
\widetilde{T}=\frac{\widetilde{\gamma}}{\widetilde{\rho}_{p}}\left(\bar{E}-\frac{1}{2} \bar{\rho} \widetilde{u}_{i} \widetilde{u}_{i}-\frac{1}{2} \tau_{i i}\right) \\
+\frac{1}{\bar{\rho}}\left[(\widetilde{\gamma}-1) \widetilde{m}\left(\frac{1}{m_{h}}-\frac{1}{m_{l}}\right)-\frac{\widetilde{\gamma}}{\widetilde{c}_{p}}\left(c_{p_{h}}-c_{p_{l}}\right)\right] \phi^{T \psi},
\end{gathered}
$$

with the filtered specific heat, molecular weight and specific heat ratio of the mixture defined as

$$
\widetilde{c}_{p}=c_{p_{h}} \widetilde{\psi}+c_{p_{l}}(1-\widetilde{\psi}), \quad \frac{1}{\widetilde{m}}=\frac{\widetilde{\psi}}{m_{h}}+\frac{1-\widetilde{\psi}}{m_{l}}, \quad \widetilde{\gamma}=\frac{\widetilde{c}_{p}}{\widetilde{c}_{p}-\mathfrak{R} / \widetilde{m}} .
$$

In the above expressions, $c_{p_{h}}$ (respectively $c_{p_{l}}$ ) and $m_{h}$ (respectively $m_{l}$ ) are the specific heat and molecular weight of the pure heavy (respectively light) fluid, and $\mathfrak{R}$ is the universal ideal gas constant. The current simulations employ $\mathrm{SF}_{6}$ and air as the heavy and light gases, whose properties are summarized in table 2. The Atwood ratio for this gas combination is $A \approx 0.67$. Ahead of the incident shock wave, the initial pressure and temperature are set to $p_{0}=23 \mathrm{kPa}$ and $T_{0}=286 \mathrm{~K}$, for which the pure $\mathrm{SF}_{6}$ and air densities are respectively $\rho_{h} \approx 1.413 \mathrm{~kg} \mathrm{~m}^{-3}$ and $\rho_{l} \approx 0.279 \mathrm{~kg} \mathrm{~m}^{-3}$, and the kinematic viscosity of the mixture $\widetilde{v} \equiv \bar{\mu} / \bar{\rho} \approx 1.6 \times 10^{-5} \mathrm{~m}^{2} \mathrm{~s}^{-1}$ when $\widetilde{\psi}=0.5$.

The dependence of the dynamic viscosity $\bar{\mu}$, thermal conductivity $\bar{\kappa}$ and mass diffusion coefficient $\widetilde{D}$ of the mixture on the filtered temperature and mass fraction are described well by Reid, Prausnitz \& Polling (1987).

The effect of the smallest, unresolved or subgrid scales (SGS) on the resolved scales is formally represented by the subgrid stress tensor $\tau_{i j}$ in $(2.5 b)$, the turbulent temperature flux $q_{j}^{T}$ and subgrid interdiffusional enthalpy flux $q_{j}^{T \partial \psi}$ in $(2.5 c)$, the subgrid scalar flux $q_{j}^{\psi}$ in $(2.5 d)$, and the temperature-scalar correlation $\phi^{T \psi}$ in (2.7). These quantities are computed locally using a distribution of stretching vortices as an explicit representation of small-scale dynamics (Misra \& Pullin 1997) that are approximate solutions of the Navier-Stokes equations (Lundgren 1982). In particular, the subgrid kinetic energy is modelled assuming an energy spectrum of subgrid vortices of the stretched-spiral type that can be computed explicitly from resolvedscale quantities and $\widetilde{v}$. The stretched-vortex SGS model has been recently extended 
to multicomponent, compressible flows by Lombardini et al. (2011) who provide a detailed expression for each subgrid term to model. We emphasize that this model explicitly depends on the kinematic viscosity of the mixture, and on no other form of viscosity.

In what follows, Favre-filtered quantities are identified with resolved-scale quantities computed in the LES, so that overbars and tildes will be omitted.

\subsection{Computational method}

A weighted essentially non-oscillatory (WENO) shock-capturing method is used to capture discontinuities such as the incident and transmitted shock waves, but the scheme dynamically reverts to a low-numerical dissipation, tuned centre-difference (TCD) scheme away from the shocks (Hill \& Pullin 2004; Lombardini 2008). The TCD scheme is optimal for the numerical computation of turbulent mixing as it minimizes the truncation errors in LES (Ghosal 1996). For the present problem, once the transmitted shock has exited the computational domain, only the TCD stencil remains used in the entire domain. The hybrid WENO-TCD method has been augmented by an intelligent switching algorithm based on Lax entropy conditions (Lombardini 2008), and tested in a variety of compressible problems involving shocks of varying strengths and density interfaces (e.g. Lombardini \& Pullin 2009).

Kosovic, Pullin \& Samtaney (2002) demonstrated the efficacy of two explicit SGS models, one of which is the stretched-vortex model, combined with compact difference schemes to reproduce the right dissipation rate of turbulent kinetic energy (TKE) predicted by the direct numerical simulation (DNS) of the decaying compressible turbulence test by Samtaney, Pullin \& Kosovic (2001). Similar results were achieved with the WENO-TCD scheme (Hill \& Pullin 2004). Using the same SGS model and the Fourier pseudospectral method, Chung \& Pullin (2010) performed DNS and LES of statistically stationary buoyancy-driven turbulent mixing, showing that both the resolved-scale and SGS-extended components of the LES velocity spectra and velocity-density cospectra accurately capture important features of the DNS. Comparisons between LES and DNS initiated by the Taylor-Green vortex (Drikakis et al. 2007) demonstrated that a variety of explicit SGS models and implicit LES models (Grinstein, Margolin \& Rider 2007) can consistently capture the physics of turbulence decay.

Since LES at very high Reynolds numbers using low-numerical-dissipation discretizations provides negligible numerical stabilization of nonlinear instabilities, momentum, energy and scalar convective terms have been written in a skew-symmetric form adapted to compressible flows (Blaisdell 1991; Honein \& Moin 2004). Lownumerical dissipation schemes for purely convective problems also impose particular temporal stability requirements that justify the use of the optimal third-order strong stability-preserving Runge-Kutta scheme of Gottlieb, Shu \& Tadmor (2001) as a reliable time-marching method.

\section{Evolution to fully developed turbulence}

In this section, we propose a method to determine for what range of $M_{I}$, as discussed in $\S 2$, the flow evolves to a fully developed turbulent state.

\subsection{Input kinetic energy at an impulsively accelerated, diffuse, perturbed interface}

Following Saffman \& Meiron (1989), we first evaluate the kinetic energy created by impulsive acceleration of an incompressible continuously stratified fluid for small, 
multimode density perturbations. The density interface extends transversally with spatial lengths $L_{y}=L_{z} \equiv L$, and the unperturbed density profile has a characteristic length scale $L_{\rho}$ in the $x$-direction. In the frame of the accelerated interface, the kinetic energy (per unit volume) can be defined by

$$
\mathscr{K}_{I}=\frac{1}{2} \int_{-L / 2}^{L / 2} \int_{-L / 2}^{L / 2} \int_{-\infty}^{\infty}\left(u_{x}^{\prime 2}+u_{y}^{\prime 2}+u_{z}^{\prime 2}\right) \frac{\mathrm{d} x}{L_{\rho}} \frac{\mathrm{d} y}{L} \frac{\mathrm{d} z}{L} .
$$

From Saffman \& Meiron (1989), it can be easily shown that, for small-amplitude perturbations, and in the diffuse interface approximation $k_{0}^{2} L_{\rho}^{2} \gg 1$,

$$
u_{x}^{\prime 2}+u_{y}^{\prime 2}+u_{z}^{\prime 2} \approx\left(\Delta u \frac{\rho^{\prime}}{\bar{\rho}}\right)^{2}
$$

where

$$
\begin{gathered}
\bar{\rho}(\zeta)=\widetilde{\rho}(1+A \tanh \zeta) \quad \text { with } \widetilde{\rho} \equiv \frac{\rho_{h}+\rho_{l}}{2}, \quad \zeta \equiv \frac{x}{L_{\rho}}, \\
\rho^{\prime}(\zeta, y, z)=\varepsilon \widetilde{\rho} g(\zeta) \quad \text { with } \varepsilon \equiv \frac{2 \xi(y, z)}{L_{\rho}} A \ll 1, \quad g(\zeta) \equiv \frac{1}{2} \operatorname{sech}^{2} \zeta,
\end{gathered}
$$

are the modelled unperturbed density distribution and density perturbation. Equation (3.1) becomes

$$
\mathscr{K}_{I} \approx \frac{2 \Delta u^{2} A^{2}}{L_{\rho}^{2} L^{2}} \int_{-\infty}^{\infty} \frac{g^{2}}{\bar{\rho}^{2}} \mathrm{~d} \zeta \int_{-L / 2}^{L / 2} \int_{-L / 2}^{L / 2} \xi^{2} \mathrm{~d} y \mathrm{~d} z .
$$

Applying this result to the interface perturbation defined in $\S 2.2$ leads to

$$
\mathscr{K}_{I}=\left(\frac{\eta_{0}}{L_{\rho}} \frac{A}{2 \sqrt{1-A^{2}}} \Delta u\right)^{2} .
$$

The equivalent single-mode perturbation defined by (2.3) naturally provides the same estimate of the input kinetic energy. To compare, the input kinetic energy for an impulsively accelerated sharp interface with a similar perturbation shape would be given by

$$
\mathscr{K}_{I}^{\text {sharp }} \approx \frac{1}{2}\left(k_{0} \eta_{0} A \Delta u\right)^{2} \quad \text { for } k_{0}^{2} L_{\rho}^{2} \ll 1,
$$

where we recognize Richtmyer's impulsive growth rate. We observe that the kinetic energy is decreased as the width of the initial unperturbed density distribution is increased (Saffman \& Meiron 1989):

$$
\frac{\mathscr{K}_{I}}{\mathscr{K}_{I}^{\text {sharp }}}=\frac{1}{2\left(1-A^{2}\right)} \frac{1}{k_{0}^{2} L_{\rho}^{2}} \ll 1 \quad \text { as } k_{0}^{2} L_{\rho}^{2} \gg 1 \text {. }
$$

\subsection{Definition of velocity fluctuations, Reynolds numbers and length scales}

For the perturbed, accelerated interface of characteristic length scale $\eta_{0}$, we define a characteristic velocity fluctuation $u_{I}^{\prime}$ based on the input kinetic energy $\mathscr{K}_{I}$, an input Reynolds number based on $u_{I}^{\prime}$ and $\eta_{0}$, and an input Taylor-scale Reynolds number $R e_{\lambda_{I}}$ as

$$
u_{I}^{\prime}=\sqrt{\frac{2}{3} \mathscr{K}_{I}}, \quad R e_{I}=\frac{u_{I}^{\prime} \eta_{0}}{v}, \quad \operatorname{Re}_{\lambda_{I}}=\sqrt{10 R e_{I}}
$$



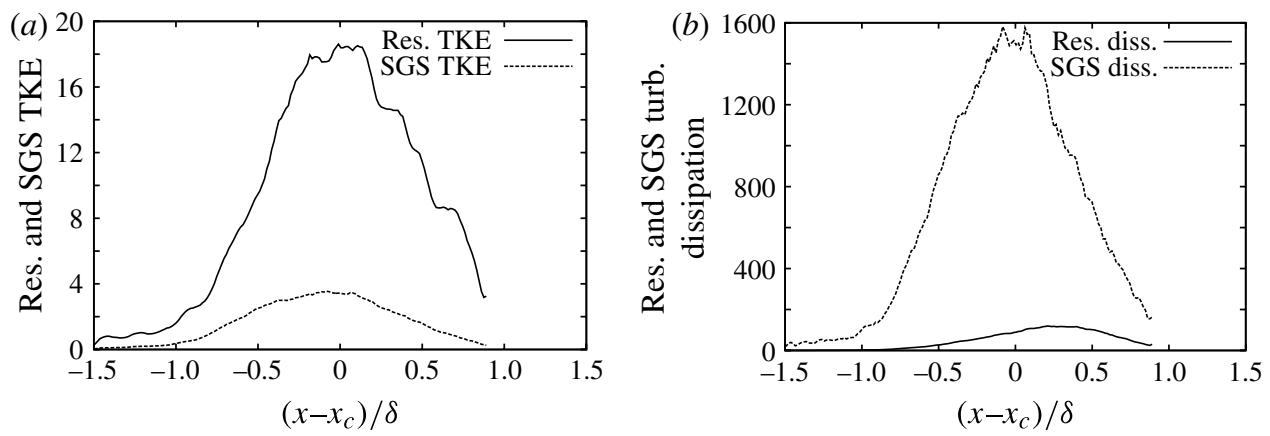

FIGURE 4. Plane-averaged resolved/subgrid TKE $(a)$ and energy dissipation rate $(b)$ profiles at $t / t_{M_{I}} \approx 800$, in the case $M_{I}=5.0$.

$\begin{array}{lccccc}M_{I} & 1.05 & 1.25 & 1.56 & 3.0 & 5.0 \\ \mathscr{K}_{I}\left(\mathrm{~m}^{2} \mathrm{~s}^{-2}\right) & 27 & 598 & 2176 & 24931 & 86380 \\ R e_{I} & 1784 & 8422 & 16067 & 54388 & 101238 \\ \operatorname{Re}_{\lambda_{I}} & 134 & 290 & 401 & 737 & 1006\end{array}$

TABLE 3. For each $M_{I}$, input kinetic energy $\mathscr{K}_{I}$, Reynolds number $R e_{I}$ and Taylor-scale Reynolds number $R e_{\lambda_{I}}$ based on an impulsive deposition of baroclinic energy at a diffuse interface.

In obtaining (3.8c) isotropy has been assumed together with the further assumption that the energy dissipation rate is constant and independent of the Reynolds number when $\operatorname{Re}_{I} \gg 1$. Using (3.5),

$$
\operatorname{Re}_{\lambda_{I}}=\left(\frac{10}{\sqrt{6}} \frac{\eta_{0} \Delta u}{v} \frac{\eta_{0}}{L_{\rho}} \frac{A}{\sqrt{1-A^{2}}}\right)^{1 / 2},
$$

which depends on the initial parameters only. Table 3 lists the values of $\mathscr{K}_{I}, R_{I}$ and $R e_{\lambda_{I}}$ computed for the experimental conditions chosen according to (3.5), (3.8) and (3.9), where $\eta_{0}$ and $A$ are identified with their post-shock equivalent $\eta_{0^{+}}$and $A^{+}$, because the model considered here portrays the impulsively accelerated flow, immediately following the initial shock refraction. The interface is initially diffuse, with $k_{0}^{2} L_{\rho}^{2} \approx 6$.

Similarly, we evaluate the r.m.s. velocity fluctuation level and Taylor-scale Reynolds number at time $t$ and on the transverse plane $x=x_{c}$, classically defined for isotropic turbulence as

$$
u^{\prime}=\sqrt{\frac{2}{3}\left(\left\langle\mathscr{K}_{\text {res }}\right\rangle+\left\langle\mathscr{K}_{\text {sgs }}\right\rangle\right)}, \quad \operatorname{Re}_{\lambda}=u^{\prime 2} \sqrt{\frac{15}{\left(\left\langle\varepsilon_{\text {res }}\right\rangle+\left\langle\varepsilon_{\text {sgs }}\right\rangle\right)\langle v\rangle}},
$$

where the resolved and subgrid components of the plane-averaged TKE, $\left\langle\mathscr{K}_{\text {res }}\right\rangle$ and $\left\langle\mathscr{K}_{\text {sgs }}\right\rangle$, and energy dissipation rate, $\left\langle\varepsilon_{\text {res }}\right\rangle$ and $\left\langle\varepsilon_{s g s}\right\rangle$, and the plane-averaged kinematic viscosity $\langle v\rangle$ of the mixture are directly measured from the computations (Lombardini et al. 2011). Typical plane-averaged profiles of the resolved and subgrid TKE and energy dissipation rate are shown in figure 4. In particular, the subgrid energy transfer off the grid is much larger (by a factor of about 10) than the (resolved) viscous 
dissipation, implying that most of the dissipation is provided by the subgrid activity as commonly observed in LES of turbulent flows. From $u^{\prime}$, a turbulent Mach number at the mixing-layer centre is given by $M_{t}=u^{\prime} /\langle c\rangle$, with $c$ the local sound speed of the mixture. To account for the inhomogeneity of the flow, directional Taylor-scale Reynolds numbers are defined by

$$
\operatorname{Re}_{\lambda_{\alpha}}=\frac{\left\langle u_{\alpha}^{\prime 2}\right\rangle}{v \sqrt{\left\langle\left(\partial u_{\alpha}^{\prime} / \partial x_{\alpha}\right)^{2}\right\rangle}}, \quad \alpha=x, y, z .
$$

Expecting statistical isotropy in the transverse directions, we define a single transverse Taylor-scale Reynolds number by $R e_{\lambda_{y z}}=\left(R e_{\lambda_{y}}+R e_{\lambda_{z}}\right) / 2$.

The Kolmogorov scale $\lambda_{K}$, characterizing the very smallest, dissipative eddies, and an inner viscous scale $\lambda_{v}$, corresponding to the wavenumber where normalized energy spectra deviate from a constant power law, i.e. the high-wavenumber end of the inertial subrange $k_{v} \lambda_{K} \approx 1 / 8$ (e.g. see compilation of previous experimental work taken from Chapman 1979 and Saddoughi \& Veeravalli 1994), are evaluated at $t$ and $x=x_{c}$ as

$$
\lambda_{K}=\left(\frac{\langle v\rangle^{3}}{\left\langle\varepsilon_{\text {res }}\right\rangle+\left\langle\varepsilon_{\text {sgs }}\right\rangle}\right)^{1 / 4}, \quad \lambda_{\nu}=16 \pi \lambda_{K} \approx 50 \lambda_{K} .
$$

At the low-wavenumber end of the inertial subrange, the (longitudinal) integral length scale $\ell$ can be assimilated to a dissipation length $\ell_{\varepsilon}$, or to the width $\ell_{\psi}$ of the autocorrelation function for the mass fraction $\psi$ (density or components of the velocity field could also be used), related to the dominant wavelength of the spectrum $E_{\psi}$ of the fluctuations $\psi^{\prime}$ (taken at time $t$ at the plane $x=x_{c}$ ):

$$
\ell_{\varepsilon}=\frac{u^{\prime 3}}{\left\langle\varepsilon_{r e s}\right\rangle+\left\langle\varepsilon_{s g s}\right\rangle}, \quad \ell_{\psi}=2 \pi \frac{\int_{0}^{k_{\max }} E_{\psi}\left(k_{r} ; x_{c}, t\right) / k_{r} \mathrm{~d} k_{r}}{\int_{0}^{k_{\max }} E_{\psi}\left(k_{r} ; x_{c}, t\right) \mathrm{d} k_{r}} .
$$

For isotropic turbulence, the ratio of the inner viscous scale to the integral scale $\ell_{\varepsilon}$ is related to the Taylor-scale Reynolds number as

$$
\frac{\ell_{\varepsilon}}{\lambda_{v}}=\frac{15^{-3 / 4}}{50} R e_{\lambda}^{3 / 2}
$$

and therefore the higher $R e_{\lambda}$, the wider is the inertial subrange.

\subsection{Discussion}

We first plot in figure 5 the evolution of the isotropic $R e_{\lambda}(t)$ defined by $(3.10 b)$, and empirically scaled by $R e_{\lambda_{I}}$ given by (3.9). For this plot, an appropriate time scale $t_{M_{I}}$ was found as

$$
\frac{1}{t_{M_{I}}}=\left(\frac{\Delta u}{a_{0}}\right)^{-1 / 2} k_{0} A^{+} \Delta u,
$$

where $\left(k_{0} A^{+} \Delta u\right)^{-1}$ is the characteristic time scale of the small-amplitude regime (Richtmyer 1960), and $\left(\Delta u / a_{0}\right)^{-1 / 2}$ is a function of the incident Mach number displayed in figure $6, a_{0}$ being the sound speed of the unshocked $\mathrm{SF}_{6}$. It is interesting to notice that this factor is similar to the one appearing in the empirical post-reshock growth rate (5.3) of Lombardini et al. (2011). Figure 6 suggests that there is a lowand a high-Mach-number regime affecting the turbulent evolution of the mixing layer 


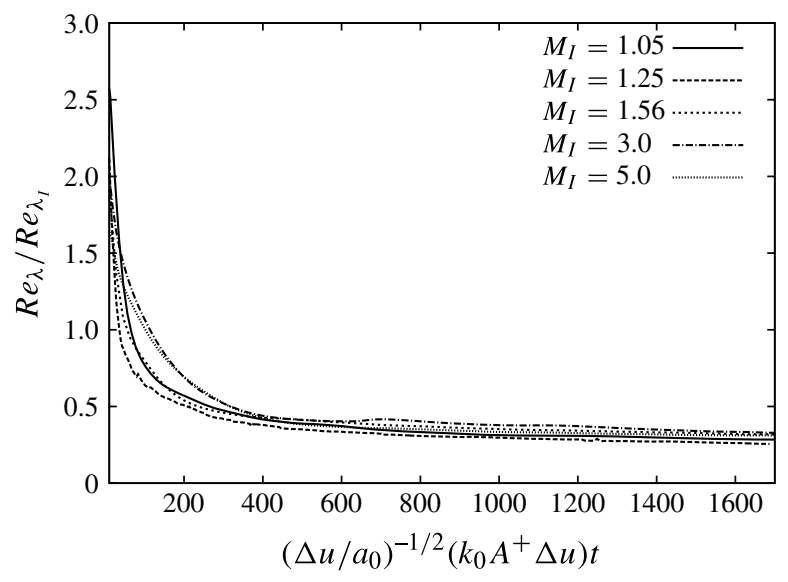

FIGURE 5. Evolution of the Taylor-scale Reynolds number (evaluated at the mixing-zone centre).

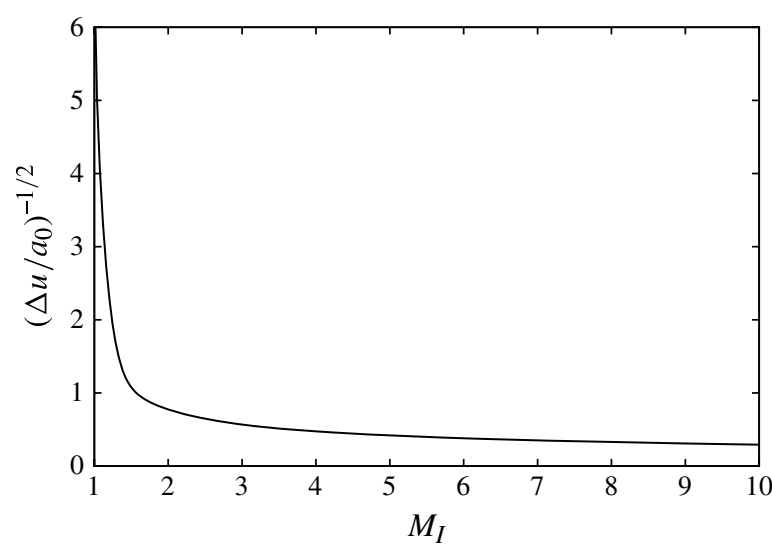

FIGURE 6. Mach-number function used in (3.15), derived by solving the one-dimensional shock-interface interaction. It can be shown that $\Delta u=b\left(M_{I}\right)\left(M_{I}-1\right)$ where $b(M)$ is a smooth, bounded function of $M_{I}$ that tends to some constants $B_{0}\left(\gamma_{1}, \gamma_{2}, A\right)$ as $M \rightarrow 1^{-}$and $B_{\infty}\left(\gamma_{1}, \gamma_{2}, A\right)$ as $M \rightarrow \infty$.

that Richtmyer's impulsive model alone cannot capture. As shown in figure 5 and reported in table 4, the simulations indicate that, for late times, when an inertial subrange has possibly been formed, $R e_{\lambda}$ slowly decays, approaching the value

$$
R e_{\lambda} \approx \mathscr{C} R e_{\lambda_{I}}
$$

with $\mathscr{C} \approx 0.3$ at $t / t_{M_{I}} \approx 1600$.

The late-time dimensionless wavenumbers associated with the integral lengths $\ell_{\psi}$ and $\ell_{\varepsilon}$, given by (3.13), and the viscous lengths $\lambda_{v}$ and $\lambda_{K}$, defined by (3.12), are reported in table 4 , and sketched in figure 7 for the particular case $M_{I}=3.0$. High values of $\lambda_{K}$ justify a posteriori the use of LES. As attested by the ratio $k_{v} / k_{\varepsilon}$, there exists an incident Mach number, $M_{I} \approx 3.0$, above which sufficient scale separation is achieved (a decade or more in scale separation between $\ell_{\varepsilon}$ and $\ell_{v}$ ), i.e. $R e_{\lambda} \gtrsim 250$. The calculations of $k_{\nu} / k_{\varepsilon}$ and $R e_{\lambda}$ are consistent with the relationships (3.13a) and (3.14). 


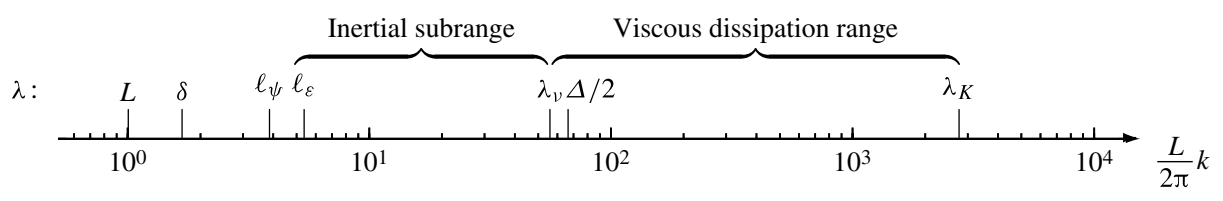

FIGURE 7. Important resolved ( $\lambda<\Delta / 2$, the Nyquist wavelength) and unresolved $(\lambda>\Delta / 2)$ length scales, and corresponding wavenumbers, for the case $M_{I}=3.0$ at $t / t_{M_{I}} \approx 1600$, for which $\operatorname{Re}_{\lambda} \approx 250$ (see table 4).

$\begin{array}{lccccc}M_{I} & 1.05 & 1.25 & 1.56 & 3.0 & 5.0 \\ R e_{\lambda} & 38 & 76 & 129 & 248 & 312 \\ R e_{\lambda} / R e_{\lambda_{I}} & 0.29 & 0.26 & 0.32 & 0.33 & 0.31 \\ (L / 2 \pi) k_{\psi} & 4.8 & 4.8 & 4.5 & 3.9 & 3.9 \\ (L / 2 \pi) k_{\varepsilon} & 14.1 & 9.5 & 6.7 & 5.4 & 5.2 \\ (L / 2 \pi) k_{\nu} & 8.7 & 16.4 & 25.6 & 55.2 & 75.4 \\ (L / 2 \pi) k_{K} & 435 & 822 & 1280 & 2760 & 3772 \\ k_{\nu} / k_{\varepsilon} & 0.62 & 1.74 & 3.83 & 10.3 & 14.5 \\ M_{t} & 0.001 & 0.002 & 0.003 & 0.005 & 0.006 \\ \operatorname{Re}_{\lambda_{x}} / \operatorname{Re}_{\lambda_{y z}} & 1.47 & 1.43 & 1.36 & 1.50 & 1.48\end{array}$

TABLE 4. For each $M_{I}$, Taylor-scale Reynolds number $R e_{\lambda}$, dimensionless wavenumbers associated with the dominant wavelength $\ell_{\psi}$, the dissipation length $\ell_{\varepsilon}$, the inner viscous scale $\lambda_{v}$ and the Kolmogorov scale $\lambda_{K}$, turbulent Mach number $M_{t}$, and directional Taylor-scale Reynolds numbers $R e_{\lambda_{\alpha}}$ computed at the late time $t / t_{M_{I}} \approx 1600$ across the mixing-layer centre-plane. $(L / 2 \pi) k_{\max }=64$ is the maximum resolved wavenumber in the present LES.

From the empirical observation (3.16) and using the model (3.9), $R e_{\lambda} \gtrsim 250$ leads to the following approximate criterion for assessing the existence of a late-time, fully developed inertial subrange, given the initial parameters of the problem:

$$
\frac{\eta_{0^{+}} \Delta u}{v} \frac{\eta_{0^{+}}}{L_{\rho}} \frac{A^{+}}{\sqrt{1-A^{+^{2}}}} \gtrsim \frac{1.53 \times 10^{4}}{\mathscr{C}^{2}} \text {. }
$$

Observe that, within the initially diffuse interface approximation $k_{0}^{2} L_{\rho}^{2} \gg 1$, the lefthand side of (3.17) is independent of the dominant wavelength $\lambda_{0}$, whereas the constant $\mathscr{C}$ formally depends on the two dimensionless parameters $A$ and $\eta_{0}^{+} / \lambda_{0}$, which are fixed in the present study.

Excluding the early stages of the mixing-layer evolution in the vicinity of the shock-interface interaction, compressibility effects in the turbulence are not large even for very high incident shock Mach numbers, as shown by the low values of the turbulent Mach number $M_{t}$ measured at the mixing-zone centre (table 4). The weakly compressible nature of the turbulence is a well-known characteristics of shock-driven mixing (e.g. see Hill et al. 2006). The initial shock-interface interaction, as well as the subsequent reverberations between the deformed front of the refracted (reflected and transmitted) waves and the accelerated interface, are inherently compressible effects and form the principal mechanisms for the deposition of vorticity in the perturbed 


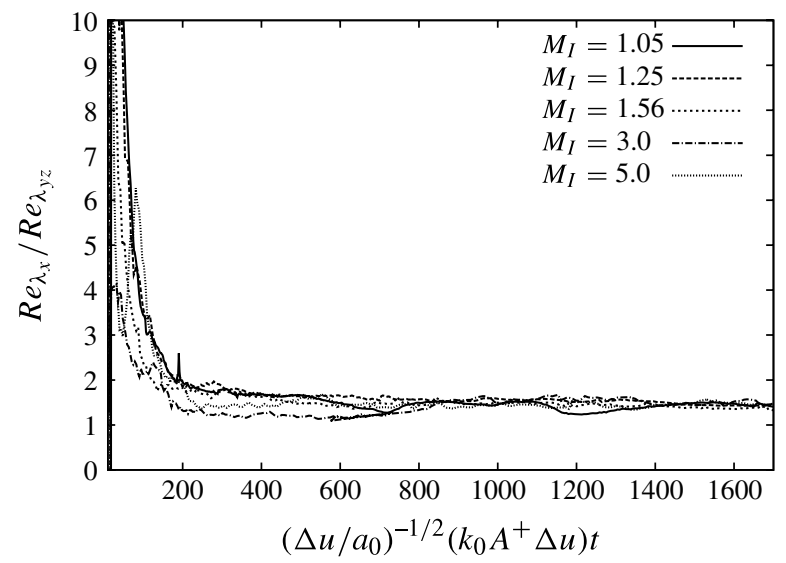

FIGURE 8. Taylor-scale-based anisotropy measure vs time (directional Taylor-scale Reynolds numbers are evaluated at the mixing-zone centre).

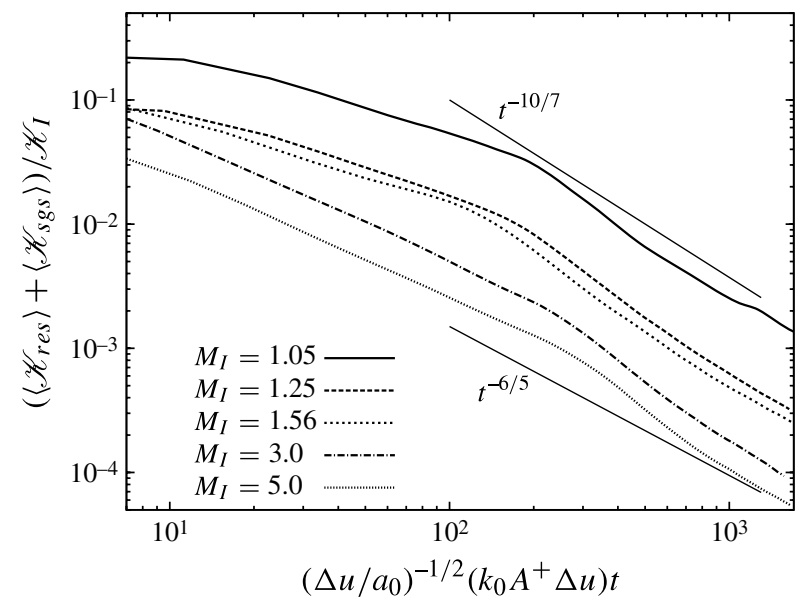

FIGURE 9. Plane-averaged (resolved + subgrid) TKE evolution on a log-log scale. The TKE is evaluated at the mixing-zone centre.

density layer. The energy is then redistributed to scales larger and smaller than the initial perturbation characteristic wavelength, evidently by means of vortex stretching, secondary baroclinic effects and bubble competition, among important causes, while compressible effects vanish.

The anisotropy in Taylor microscales is shown in figure 8, where at late times $R e_{\lambda_{x}} / R e_{\lambda_{y z}} \approx 1.5$, for any of the Mach numbers investigated.

\section{Decay of TKE}

The kinetic energy initially deposited during the shock passage is irreversibly transferred into the internal energy of the fluid by viscous action. We plot in figure 9 the evolution of the plane-averaged total TKE evaluated at the centre-plane, and scaled by $\mathscr{K}_{I}$ given by (3.5). For all Mach numbers considered, past the early-time growth of the two-fluid mixing zone $t / t_{M_{I}}>200$, the TKE decays like $t^{-n}$, with $n \approx 1.4$. For 


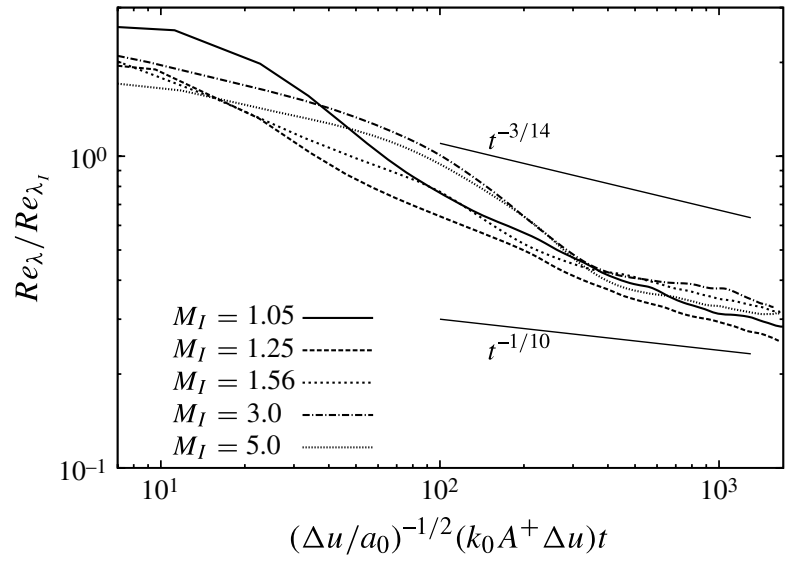

FIGURE 10. Evolution of the Taylor-scale Reynolds number on a log-log scale (plane-averages are evaluated at the mixing-zone centre).

comparison, we display the $t^{-10 / 7}$ decay typical of Batchelor turbulence (Batchelor \& Proudman 1956), as well as the $t^{-6 / 5}$ decay characteristic of Saffman turbulence (Saffman 1967). For the time range considered $n$ seems greater than $6 / 5$, and closer to $10 / 7$.

The decay exponent is usually measured from experiments of single-fluid grid turbulence, considered as a good approximation for the decay of high-Reynoldsnumber, strictly homogeneous turbulence. Lavoie, Djenidi \& Antonia (2007) showed, for different type of grids, decay exponents slightly lower than the Saffman estimate, while Krogstad \& Davidson (2010) found that the turbulence behind their grid is of the type envisaged by Saffman (1967) with $n \approx 1.13$. We recall that, in turbulence theory, $\mathscr{A}=\varepsilon \ell_{\varepsilon} / u^{\prime 3}$ is a numerical constant of the order of unity at high Reynolds number, i.e. independent of viscosity (Sreenivasan 1984), where the total energy dissipation rate, $\varepsilon=\left\langle\varepsilon_{\text {res }}\right\rangle+\left\langle\varepsilon_{\text {sgs }}\right\rangle$, is a surrogate for the energy flux from the large to small scales. This is naturally consistent with the definition (3.13a).

If the TKE decays like $t^{-n}$, it is easy to show that the isotropic $R e_{\lambda}$ must decay with a slower exponent of $-(n-1) / 2$. The late-time slow decay of $R e_{\lambda}$ can be seen in figure 5, or equivalently in figure 10 on a logarithmic scale, where the corresponding Saffman and Batchelor decay slopes have been included for comparison. From (3.14), a direct consequence of the slow decay of $R e_{\lambda}$ is a slow contraction of the inertial subrange. In the limit $t \rightarrow \infty$, the inertial subrange would ultimately disappear.

\section{Power spectra}

For any quantity $Q(x, y, z ; t)$, a radial energy spectrum $E_{Q}\left(k_{r} ; x, t\right)$ of the fluctuation $Q^{\prime}(x, y, z ; t)$ can be computed at the plane located at $x$ and at time $t$ by applying the Fourier transform of $Q^{\prime}$, multiplying by its complex conjugate, and summing over the shell associated with each radial wavenumber bin.

Figure 11 depicts compensated radial power spectra $k_{r}^{-5 / 3} E_{u_{x}}$ of the axial velocity component $u_{x}$ computed across a transverse plane slicing through the mixing-layer centre $x=x_{c}$, at three different times. As the mixing layer grows, the peak in the power spectrum, initially located near the dominant wavenumber $(L / 2 \pi) k_{0}$, drifts to lower wavenumbers due to the merging of bubble structures and the generation of long-range correlations. The spectrum also expands towards higher wavenumbers 

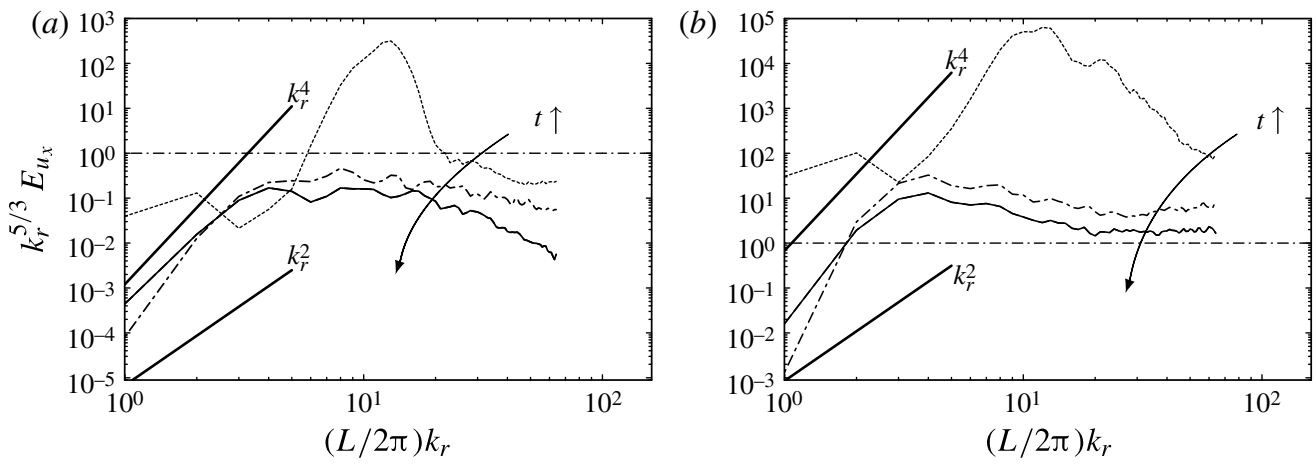

FIGURE 11. Compensated axial velocity power spectra at three different times $t / t_{M_{I}} \approx 10$ (dotted line), 800 (dash-dotted line) and 1600 (solid line). The arrow indicates the increase in time, the thin dash-dotted horizontal line the $k_{r}^{-5 / 3}$ power law, and the two thick solid segments the large-scale theoretical behaviours $E_{u_{x}}\left(k_{r} \rightarrow 0\right) \sim k_{r}^{2}$ (Saffman spectrum) and $\sim k_{r}^{4}$ (Batchelor spectrum). $M_{I}=1.05(a)$ and $M_{I}=3.0(b)$. All computed wavenumbers shown and $(L / 2 \pi) k_{\max }=64$.

due to vortex stretching. In figure 11(a), corresponding to the lowest Mach number $M_{I}=1.05$, no clear inertial subrange characteristic of fully developed turbulence is observed, even at the latest times. Similar observations were made for $M_{I}=1.25$ while the other three higher Mach number cases (e.g. as shown in figure $11(b)$ for $M_{I}=3.0$ ) show a developing range of scales following an approximate $-5 / 3$ powerlaw Kolmogorov-like scaling persistent at late times.

Plots of the $k_{r}^{2}$ - and $k_{r}^{4}$-slopes are also represented on figure 11 , indicating that, at late times and in fact for all the Mach numbers tested, the large-scale motions deviate from Saffman (1967) in which the energy spectrum $E(k) \sim k^{2}$ as $k \rightarrow 0$, and instead approach the Batchelor spectrum $E(k) \sim k^{4}$. This is consistent with the observation made in the previous section that, for the current flow, the late-time decay exponent of TKE is greater than the Saffman value 6/5, and more generally that the shock-driven turbulent flow would depart from Saffman decaying turbulence and be more of Batchelor type. This remains to be tested by experimental measurements of the development of integral velocity and length scales, $u^{\prime}$ and $\ell$, to assess whether they satisfy $u^{2} \ell^{5} \approx$ constant suggestive of Batchelor turbulence. It is also entirely possible that different types of initial perturbation shape and different Atwood ratios could produce different results.

The highest resolved wavenumbers show minor aliasing error pile-up. We recall that WENO is not used within the mixing region and no explicit filtering of any kind was performed. On the other hand, pure shock-capturing methods are intrinsically more stable than low-numerical dissipation schemes, such as TCD, but provide excessive numerical dissipation for the production of reasonable power spectra, as shown for example in the simulations of Miles et al. (2005) for similar shock-driven systems and as assessed more generally by Johnsen et al. (2010).

Figure 12 contrasts energy spectra, in log-linear scale, at early and late times. The velocity spectra are multiplied by $k_{r}$ so that equal areas under the curve $k_{r} E_{u_{x}}\left(k_{r}\right)$ have equal energy. The corresponding energy spectra are then normalized by the input kinetic energy of a single velocity component, $\mathscr{K}_{I} / 3$, and scaled by different numerical factors so that they can be compared on the same plot, the total kinetic energy being dissipated. 

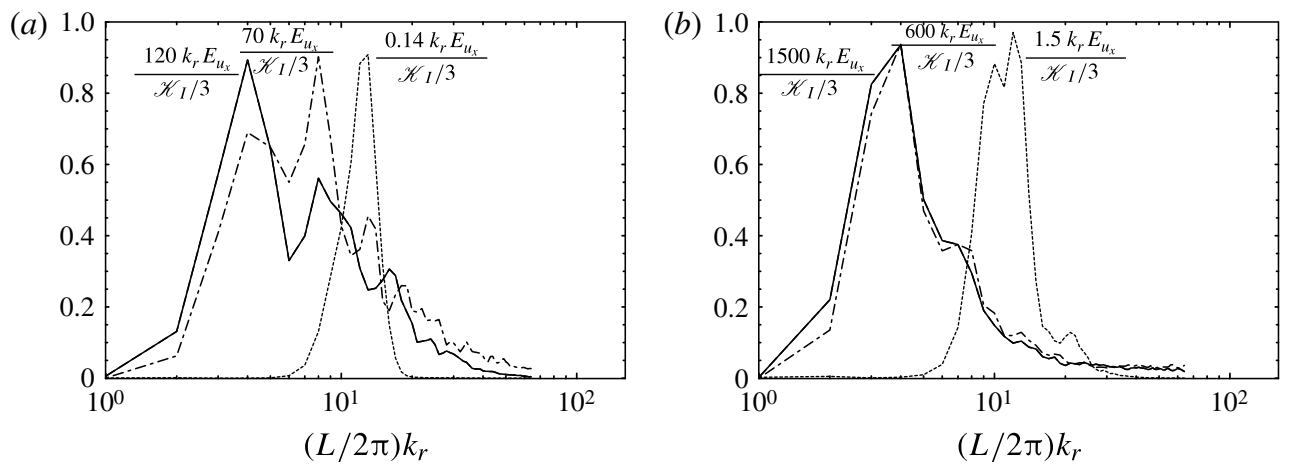

FIGURE 12 . Energy spectra $k_{r} E_{u_{x}}\left(k_{r}\right)$ for the axial velocity (normalized by $\mathscr{K}_{I} / 3$ ) at three different times $t / t_{M_{I}} \approx 10$ (dotted line), 800 (dash-dotted line) and 1600 (solid line). Note the scaling of $E_{u_{x}} . M_{I}=1.05(a)$ and $M_{I}=3.0(b)$. All computed wavenumbers shown and $(L / 2 \pi) k_{\max }=64$.
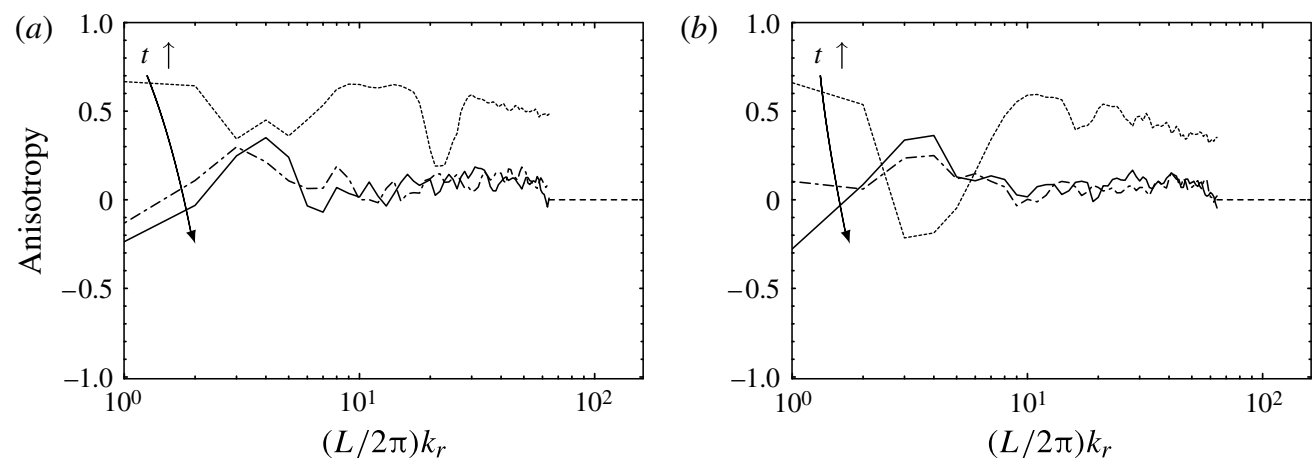

FIGURE 13. Anisotropy measure of the velocity power spectra, $E_{u_{x}} /\left(E_{u_{x}}+E_{u_{y}}+E_{u_{z}}\right)-1 / 3$, at three different times $t / t_{M_{I}} \approx 10$ (dotted line), 800 (dash-dotted line) and 1600 (solid line). $M_{I}=1.05(a)$ and $M_{I}=3.0(b)$. All computed wavenumbers shown and $(L / 2 \pi) k_{\max }=64$.

In addition to the axial velocity spectra, a spectral measure of the anisotropy at the different resolved scales is displayed in figure 13 for $M_{I}=1.05$ and 3.0 (although similar results are observed at the three other Mach numbers). It indicates that, at early times, the axial velocity power spectrum contains at almost every scale more than a third of the total energy (i.e. the energy due to axial and transverse components of the velocity). As the mixing layer grows, an isotropization of the flow is evident although perfect isotropy in the sense of TKE is never completely reached, even at the highest $M_{I}$ considered. This spectral measure of the anisotropy, which solely relies on the components of the TKE, must be complemented with the anisotropic Taylor-microscale measure described in $\S 3.3$, which is based on both TKE and energy dissipation rate.

\section{Mixing statistics}

\subsection{Mixing-layer width}

The mixing-layer width can be defined by the integral measure

$$
\delta(t)=4 \int_{-\infty}^{\infty}(1-\langle\psi\rangle)\langle\psi\rangle \mathrm{d} x,
$$




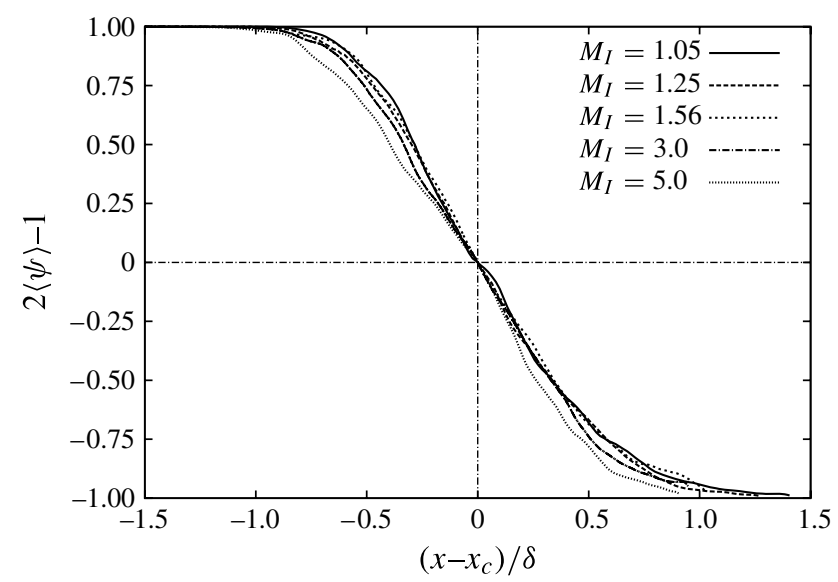

FIGURE 14. Plane-averaged mass fraction $\langle\psi\rangle$ profile at $t / t_{M_{I}} \approx 1600$. The shifted axial coordinate $x-x_{c}$ is normalized by the mixing-layer width $\delta(t)$ associated with each Mach number.
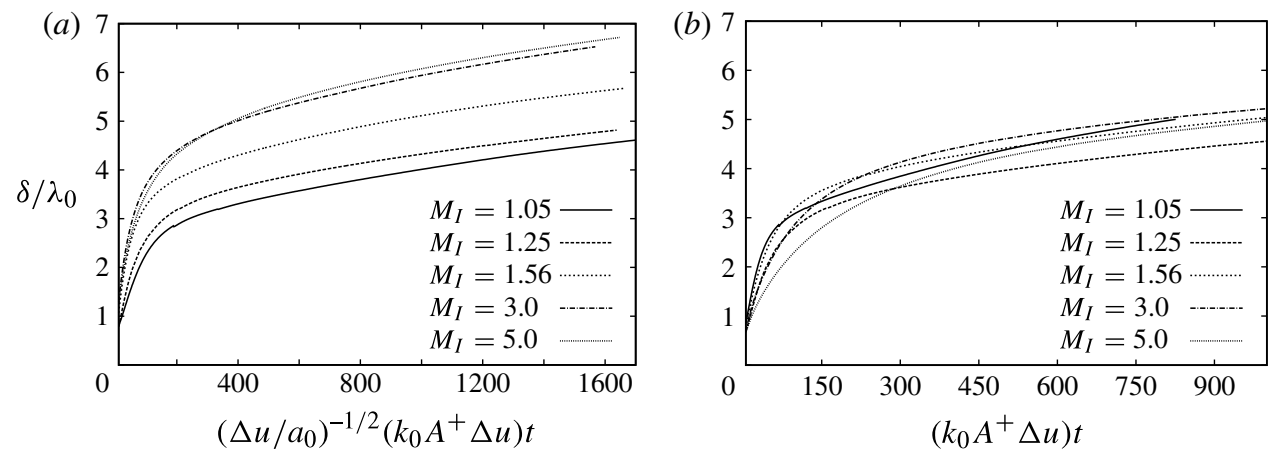

FIGURE 15. Mixing-layer width evolution. Time is either normalized by (a) $t_{M_{I}}$ given by (3.15), or (b) Richtmyer's time scale $1 /\left(k_{0} A^{+} \Delta u\right)$.

where $\psi$ is defined in $(2.5 d)$ and plane-averaged profiles $\langle\psi\rangle(x, t)$ are shown for example in figure 14. To understand this definition, consider a smooth transition from air $(\langle\psi\rangle=0)$ to $\mathrm{SF}_{6}(\langle\psi\rangle=1)$ modelled by a tanh profile of characteristic width $\delta$ centred at the location $x_{c}$ defined by $\langle\psi\rangle\left(x_{c}, t\right)=0.5$. The length $\delta$ is recovered when performing the integral in (6.1). Based on the same symmetric tanh profile, $\delta$ is $\approx 2.3$ times smaller than $\delta_{1 \%}$, another common measure of the mixing-layer width defined as the difference between the spike location $x_{S}$, for which $\langle\psi\rangle\left(x_{S}, t\right)=0.01$, and the bubble location $x_{B}$, for which $\langle\psi\rangle\left(x_{B}, t\right)=0.99$.

For the five Mach numbers considered, the evolution of $\delta$ is displayed in figure 15, for two different time scalings: $t_{M_{I}}$ given by (3.15) in (a), and Richtmyer's time scale $1 /\left(k_{0} A^{+} \Delta u\right)$ in $(b)$, the latter being the natural time scale for the mixing-layer width (Jacobs \& Krivets 2005; Orlicz et al. 2009).

At late times, when the mixing-layer width compares with the initial perturbation dominant wavelength, the mixing layer grows at a slower rate and $\delta \sim t^{\theta}$ with $\theta$ in the range $\approx 0.2-0.33$, depending on $M_{I}$ (figure 16). Previous theoretical analysis found that the nonlinear mixing-layer growth is characterized by two distinct power laws, $t^{\theta_{S}}$ 


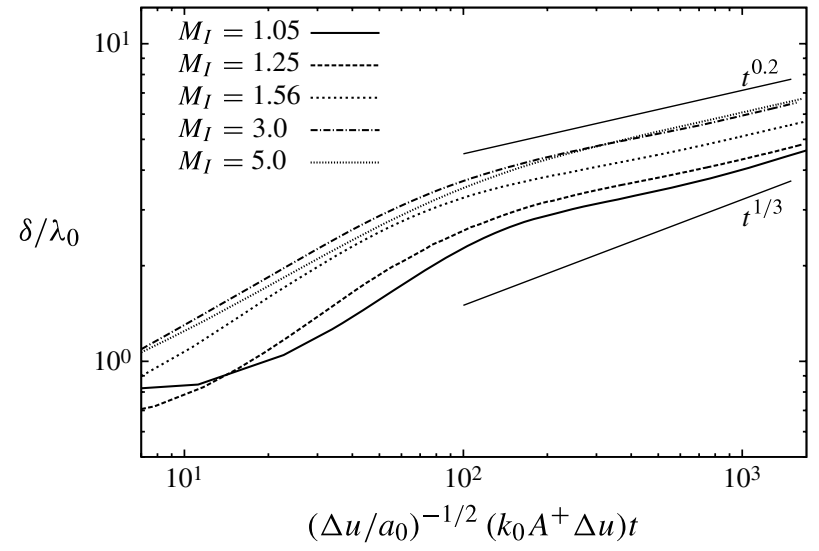

FIGURE 16. Mixing-layer width evolution on a log-log scale. Time is normalized by $t_{M_{I}}$ given by (3.15).

and $t^{\theta_{B}}$ for the spike and bubble front respectively. For example, Oron et al. (2001) used scaling arguments to show that $\theta_{S} \approx 1.7 \theta_{B}$ for $A \approx 0.7$, with $\theta_{B} \approx 0.2-0.25$. At late times, the mixing-layer width would therefore be dominated by the spike growth and $\delta \sim t^{\theta}$ with $\theta \approx 0.34-0.43$. The Linear Electric Motor experiments by Dimonte \& Schneider (2000) predicted similar power laws, but with $\theta_{S} \approx 1.44 \theta_{B}$ for $A \approx 0.7$ and $\theta_{B} \approx 0.25$, which give a late-time mixing-layer width evolving $\sim t^{0.36}$. In their analysis, Zhou and coworkers have argued however that $\theta_{B}=\theta_{S} \equiv \theta$ but with different time origin (Zhou et al. 2003) and $\theta \approx 2 / 3-7 / 12$ (Zhou 2001). Discrepancies between these published results and with the present measurements can be imputed to differences in initial perturbations and impulsive velocities investigated.

\subsection{Mixing variables}

As another interpretation of (6.1), consider the amount of mixed fluid as quantified by a passive chemical equilibrium between light and heavy fluids. The mass fraction of product is $[1-\langle\psi\rangle(x, t)] /\left[1-\langle\psi\rangle\left(x_{c}, t\right)\right]\langle\psi\rangle(x, t) /\langle\psi\rangle\left(x_{c}, t\right)$, and the width $\delta$ at time $t$ defined by (6.1) can then be interpreted as a product thickness that would result if the entrained fluids were perfectly mixed in $y$ and $z$. Moreover, the ratio

$$
\Theta(t)=\frac{\int_{-\infty}^{\infty}\langle(1-\psi) \psi\rangle \mathrm{d} x}{\int_{-\infty}^{\infty}(1-\langle\psi\rangle)\langle\psi\rangle \mathrm{d} x}
$$

defined by Youngs (1994) characterizes the relative amount of molecularly mixed fluid within the mixing layer (i.e. total product formed relative to the product that would be formed if all entrained fluid were completely mixed within each transverse plane). Another mixing variable is the effective Atwood ratio $A_{e}$ (Cook, Cabot \& Miller 2004), which we define at the centre-plane by

$$
A_{e}(t)=\frac{\sqrt{\left\langle\rho^{\prime 2}\right\rangle\left(x_{c}, t\right)}}{\langle\rho\rangle\left(x_{c}, t\right)} .
$$




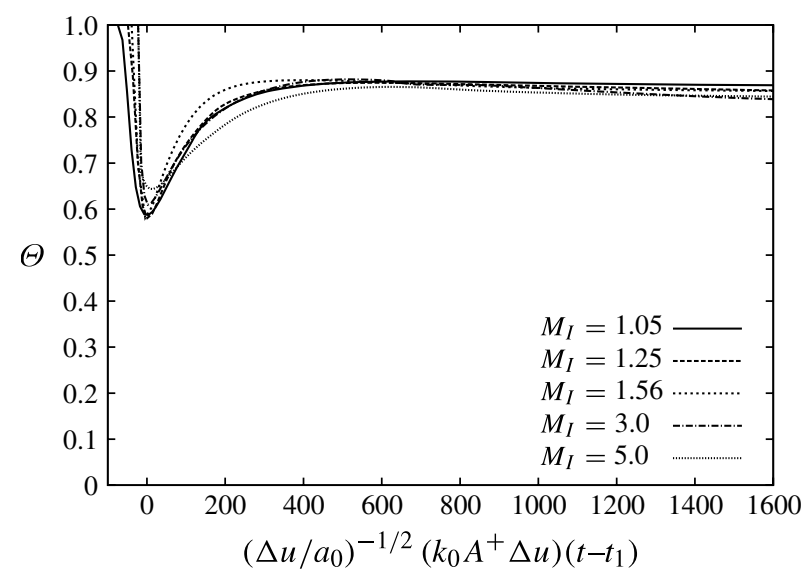

FIGURE 17. Ratio of mixed to entrained fluid $\Theta$ for the mixing region: $\Theta=1$ corresponds to completely mixed fluid (no transverse variation), whereas $\Theta=0$ corresponds to complete segregation (immiscible case).

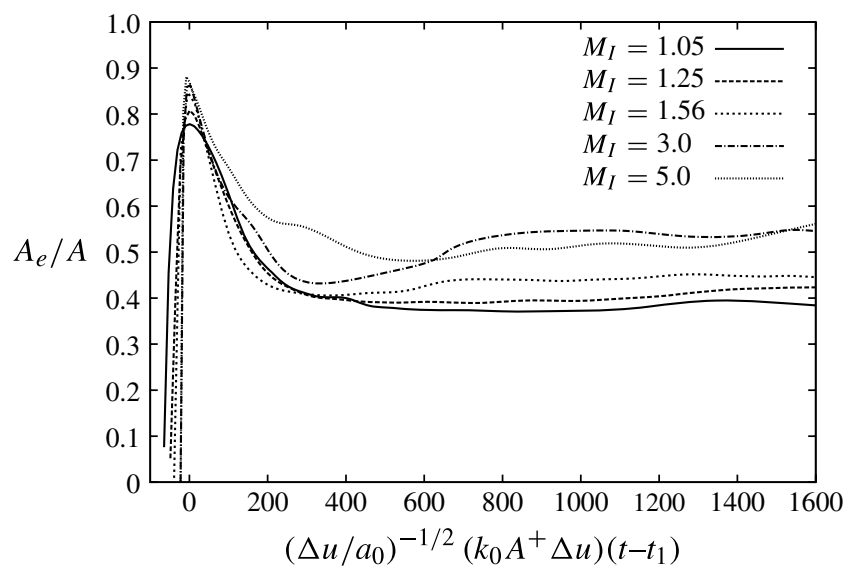

Figure 18. Effective Atwood number ratio $A_{e} / A: A_{e} / A=0$ corresponds to completely mixed fluid (no transverse variation), whereas $A_{e} / A=1$ corresponds to complete segregation (immiscible case).

The quantities $\Theta$ and $A_{e}$ complement statistics based solely on $\langle\psi\rangle$ (e.g. $\delta$ ) that cannot distinguish between fluid locally mixed for example at a fraction $\psi(x, y, z ; t)=0.5$ and unmixed fluid in equal proportions in a particular transverse plane for which $\langle\psi\rangle(x, t)=0.5$. After the shock has compressed the perturbed interface, the mixing layer is essentially characterized by small-amplitude perturbations and an inner diffuse length; therefore $\Theta$ is close to unity and $A_{e}$ reaches a minimum value (figures 17 and 18). The early growth of the initial perturbations is associated with an increase in segregation during the initial development of the flow and ends with a minimum in $\Theta$ and a peak in $A_{e}$. These extrema are reached at a time $t_{1}$ (different for each $M_{I}$ ) that was used as a time shift to obtain a better superposition of the different cases. After $t_{1}, \Theta$ starts increasing and $A_{e}$ decreasing, indicating mixing in progress (the two fluids are mixing by diffusion faster than pure fluids are coming 
into contact with the centre-plane), while the mixing-layer growth slows down. Note that the mixing activity in the LES, revealed for example by the reduction of $A_{e}$ with respect to the completely segregated state, is a result of mass diffusion by the SGS model and can be considered physical to the extent that the winding of the scalar field (i.e. mass fraction) by an elemental subgrid stretched-spiral vortex is representative of turbulent mixing processes affecting the resolved motion (Pullin 2000; Hill et al. 2006). Ultimately, $\Theta$ and $A_{e} / A$ reach nearly constant values: the two gases mix at the same final mixing parameter $\Theta \approx 0.85$, independently of $M_{I}$, while $A_{e} / A$ reaches a final value $\approx 0.4$ for low values of $M_{I}$ and $\approx 0.55$ at strong ones. Unlike figure 15 and in accordance with figure 5, figures 17 and 18 confirm that $t_{M_{I}}$, rather than $1 /\left(k_{0} A^{+} \Delta u\right)$, is a suitable time scale for characterizing the mixing process.

\section{Conclusion}

We have considered the shock-induced mixing at a heavy-light $\mathrm{SF}_{6}$-air $(A \approx 0.67)$ perturbed interface impacted by a single shock of Mach number $M_{I}=1.05,1.25$, $1.56,3.0$ or 5.0, given a similar post-shock amplitude $\eta_{0^{+}} \approx 0.25 \lambda_{0}$ for the five Mach numbers considered. The incident shock deposits baroclinic vorticity across an initially given broad spectrum of scales with peak wavelength $\lambda_{0}$ that relaxes to a state that shares some characteristics of classic incompressible decaying homogeneous turbulence for certain values of $M_{I}$. We observed

(a) the development of a $-5 / 3$ power-law inertial subrange for $M_{I} \geqslant 1.56$;

(b) a late-time scale separation $\left(R e_{\lambda} \gtrsim 250\right)$ for $M_{I} \geqslant 3.0$;

(c) a late-time quasi-isotropization of the flow in the sense of the r.m.s. fluctuations of the three velocity components;

(d) an incomplete isotropization of the flow in the sense of the Taylor directional microscales;

(e) late-time weakly compressible turbulence $\left(M_{t} \lesssim 0.01\right)$;

(f) a $t^{-n}$ late-time decay of kinetic energy with $n \approx 1.4$, resembling Batchelor grid turbulence where $E(k)(k \rightarrow 0) \sim k^{4}$; and a subsequently slow contraction of the inertial subrange (when existing);

(g) a late-time mixing at a mixing parameter $\Theta \approx 0.85$;

(h) $1 /\left(k_{0} A^{+} \Delta u\right)$ as a natural time scale for the evolution of $\delta$ only, but $t_{M_{I}}$ given by (3.15) as a more appropriate time scale to study the turbulent mixing;

(i) the existence of low- and high-Mach-number regimes (e.g. through the introduction of $t_{M_{I}}$, and according to the late-time asymptotic values of $A_{e}$ ).

While the time scale $t_{M_{I}}$ seems to characterize the weakly compressible development of the turbulent mixing rather than the early, compressible stages of the layer growth, its physical significance remains unrevealed.

A comprehensible criterion that determines whether the flow has evolved to fully developed turbulence would combine $(a)$ and $(b)$. We have proposed an empirical prediction of the scale separation $(b)$, explicitly given by (3.17), depending on the initial parameters through a modelled Taylor-scale-based Reynolds number $\operatorname{Re}_{\lambda_{I}}$ related to the input kinetic energy injected into the perturbed, diffuse layer during the initial shock refraction. Although the transition to turbulence observed seems well described by the prediction (3.17), it is possible that different Atwood ratios or different initial perturbation amplitude-to-wavelength ratios could produce different values of $\mathscr{C}$. We 
also suggest as further research the adaptation of our predictive model to gas curtain configurations.

The explicit, stretched-vortex subgrid model employed in this study, combined with a low-numerical-dissipation scheme, allowed the production of reasonable power spectra as well as the calculation of Taylor- and Kolmogorov-scale statistics, which rely on estimating the correct contribution of the unresolved scales in the flow.

\section{Acknowledgements}

This work has been supported in part by the Department of Energy under subcontract no. DE-AC52-06NA25396.

\section{REFERENCES}

Balakumar, B. J., Orlicz, G. C., Tomkins, C. D. \& Prestridge, K. P. 2008 Simultaneous particle-image velocimetry-planar laser-induced fluorescence measurements of Richtmyer-Meshkov instability growth in a gas curtain with and without reshock. Phys. Fluids 20 (12), 124103.

Batchelor, G. K. \& Proudman, I. 1956 The large-scale structure of homogeneous turbulence. Phil. Trans. R. Soc. Lond. A 248, 369-405.

BLAisDell, G. A. 1991 Numerical simulation of compressible homogeneous turbulence. PhD thesis, Stanford University.

Chapman, D. R. 1979 Computational aerodynamics development and outlook. AIAA J. 17, 1293-1313.

Chung, D. \& Pullin, D. I. 2010 Direct numerical simulation and large-eddy simulation of stationary buoyancy-driven turbulence. J. Fluid Mech. 643 279-308.

COllins, B. D. \& JACOBS, J. W. 2002 PLIF flow visualization and measurements of the Richtmyer-Meshkov instability of an air/SF 6 interface. J. Fluid Mech. 464, 113-136.

Cook, A. W., CАвот, W. \& Miller, P. L. 2004 The mixing transition in Rayleigh-Taylor instability. J. Fluid Mech. 511, 333-362.

Dimonte, G. \& Schneider, M. 2000 Density ratio dependence of Rayleigh-Taylor mixing for sustained and impulsive acceleration histories. Phys. Fluids 12 (2), 304-321.

Dimotakis, P. E. 2000 The mixing transition in turbulent flows. J. Fluid Mech. 409, 69-97.

Drikakis, D., Fureby, C., Grinstein, F. F. \& Youngs, D. 2007 Simulation of transition and turbulence decay in the Taylor-Green vortex. J. Turbul. 8 1-12.

Ghosal, S. 1996 An analysis of numerical errors in large-eddy simulations of turbulence. J. Comput. Phys. 125 (1), 187-206.

Glezer, A. 1988 The formation of vortex rings. Phys. Fluids 31 (12), 3532-3542.

Gottlieb, S., Shu, C. -W. \& TAdmor, E. 2001 Strong stability-preserving high-order time discretization methods. SIAM Rev. 43 (1), 89-112.

Grinstein, F. F., Margolin, L. G. \& Rider, W. G. 2007 Implicit Large-Eddy Simulation: Computing Turbulent Flow Dynamics. Cambridge University Press.

Hill, D. J., Pantano, C. \& Pullin, D. I. 2006 Large-eddy simulation and multi-scale modelling of Richtmyer-Meshkov instability with reshock. J. Fluid Mech. 557, $29-61$.

Hill, D. J. \& Pullin, D. I. 2004 Hybrid tuned centre-difference-WENO method for large eddy simulations in the presence of strong shocks. J. Comput. Phys. 194 (2), 435-450.

Honein, A. E. \& Moin, P. 2004 Higher entropy conservation and numerical stability of compressible turbulence simulations. J. Comput. Phys. 201 (2), 531-545.

JACOBS, J. W. \& KRIVETS, V. V. 2005 Experiments on the late-time development of single-mode Richtmyer-Meshkov instability. Phys. Fluids 17, 034105.

Johnsen, E., Larsson, J., Bhagatwala, A. V., Cabot, W. H., Moin, P., Olson, B. J., Rawat, P. S., Shankar, S. K., Suögreen, B., Yee, H. C., Zhong, X. \& Lele, S. K. 2010 Assessment of high-resolution methods for numerical simulations of compressible turbulence with shock waves. J. Comput. Phys. 229, 1213-1237. 
KonRAD, J. H. 1976 An experimental investigation of mixing in two-dimensional turbulent shear flows with applications to diffusion-limited chemical reactions. PhD thesis, California Institute of Technology.

Kosovic, B., Pullin, D. I. \& Samtaney, R. 2002 Subgrid-scale modelling for large-eddy simulations of compressible turbulence. Phys. Fluids 14 (4), 1511-1522.

Krogstad, P. Å. \& Davidson, P. A. 2010 Is grid turbulence Saffman turbulence? J. Fluid Mech. 642, 373-394.

Lavoie, P., DJenidi, L. \& Antonia, R. A. 2007 Effects of initial conditions in decaying turbulence generated by passive grids. J. Fluid Mech. 585, 395-420.

Leinov, E., Malamud, G., Elbaz, Y., Levin, L. A., Ben-Dor, G., Shvarts, D. \& Sadot, O. 2009 Experimental and numerical investigation of the Richtmyer-Meshkov instability under re-shock conditions. J. Fluid Mech. 626, 449-475.

Lombardini, M. 2008 Richtmyer-Meshkov instability in converging geometries. PhD thesis, California Institute of Technology.

Lombardini, M., Hill, D. J., Pullin, D. I. \& Meiron, D. I. 2011 Atwood ratio dependence of Richtmyer-Meshkov flows under reshock conditions using large-eddy simulations. J. Fluid Mech. 670, 439-480.

Lombardini, M. \& Pullin, D. I. 2009 Small-amplitude perturbations in the three-dimensional cylindrical Richtmyer-Meshkov instability. Phys. Fluids 21 (11), 114103.

Lundgren, T. S. 1982 Strained spiral vortex model for turbulence fine structure. Phys. Fluids 25 (12), 2193-2203.

Miles, A. R., Blue, B., Edwards, M. J., Greenough, J. A., Hansen, J. F., Robey, H. F., Drake, R. P., Kuranz, C. \& Leibrandt, D. R. 2005 Transition to turbulence and effect of initial conditions on three-dimensional compressible mixing in planar blast-wave-driven systems. Phys. Plasmas 12, 056317.

Misra, A. \& Pullin, D. I. 1997 A vortex-based model for large-eddy simulation. Phys. Fluids 9 (8), 2443-2454.

Motl, B., Oakley, J., Ranjan, D., Weber, C., Anderson, M. \& Bonazza, R. 2009 Experimental validation of a Richtmyer-Meshkov scaling law over large density ratio and shock strength ranges. Phys. Fluids 21 (12), 126102.

Orlicz, G. C., Balakumar, B. J., Tomkins, C. D. \& Prestridge, K. P. 2009 A Mach number study of the Richtmyer-Meshkov instability in a varicose, heavy-gas curtain. Phys. Fluids 21 (6), 064102.

Oron, D., Arazi, L., Kartoon, D., Rikanati, A., Alon, U. \& Shvarts, D. 2001 Dimensionality dependence of the Rayleigh-Taylor and Richtmyer-Meshkov instability late-time scaling laws. Phys. Plasmas 8 (6), 2883-2889.

Prestridge, K., Rightley, P. M., VorobiefF, P., Benjamin, R. F. \& Kurnit, N. A. 2000 Simultaneous density-field visualization and PIV of a shock-accelerated gas curtain. Exp. Fluids 29, 339-346.

Pullin, D. I. 2000 A vortex-based model for the subgrid flux of a passive scalar. Phys. Fluids $\mathbf{1 2}$ (9), 2311-2319.

Pullin, D. I., Buntine, J. D. \& SAffman, P. G. 1994 On the spectrum of a stretched spiral vortex. Phys. Fluids 6, 3010-3027.

Reid, R. C., Prausnitz, J. M. \& Polling, B. E. 1987 The Properties of Gases and Liquids. McGraw-Hill.

RichtMYeR, R. D. 1960 Taylor instability in shock acceleration of compressible fluids. Commun. Pure Appl. Maths 13, 297-319.

Rightley, P. M., Vorobieff, P., Martin, R. \& Benjamin, R. F. 1999 Experimental observations of the mixing transition in a shock-accelerated gas curtain. Phys. Fluids 11 (1), 186-200.

Saddoughi, S. G. \& Veeravalli, S. V. 1994 Local isotropy in turbulent boundary layers at high reynolds number. J. Fluid Mech. 268, 333-372.

SAfFMAn, P. G. 1967 The large-scale structure of homogeneous turbulence. J. Fluid Mech. 27, 581-593. 
SAFFMAN, P. G. \& MeIron, D. I. 1989 Kinetic energy generated by the incompressible Richtmyer-Meshkov instability in a continuously stratified fluid. Phys. Fluids A 1 (11), 1767-1771.

Samtaney, R., Pullin, D. I. \& Kosovic, B. 2001 Direct numerical simulation of decaying compressible turbulence and shocklet statistics. Phys. Fluids 13 (5), 1415-1430.

SAMTANEY, R. \& ZABUSKY, N. J. 1994 Circulation deposition on shock-accelerated planar and curved density-stratified interfaces: models and scaling laws. J. Fluid Mech. 269, 45-78.

SReenivasan, K. R. 1984 On the scaling of the turbulent energy dissipation rate. Phys. Fluids 27, $1048-1050$.

Tomkins, C., Kumar, S., Orlicz, G. \& Prestridge, K. 2008 An experimental investigation of mixing mechanisms in shock-accelerated flow. J. Fluid Mech. 611, 131-150.

Vetter, M. \& Sturtevant, B. 1995 Experiments on the Richtmyer-Meshkov instability of an air/SF 6 interface. Shock Waves 4, 247-252.

Vorobieff, P., Rightley, P. M. \& Benjamin, R. F. 1998 Power-law spectra of incipient gas-curtain turbulence. Phys. Rev. Lett. 81 (11), 2240-2243.

VorobiefF, P., Rightley, P. M. \& Benjamin, R. F. 1999 Shock-driven gas curtain: fractal dimension evolution in transition to turbulence. Physica D 133, 469-476.

Youngs, D. L. 1994 Numerical simulations of mixing by Rayleigh-Taylor and Richtmyer-Meshkov instabilities. Laser Part. Beams 12, 725-750.

ZHOU, Y. 2001 A scaling analysis of turbulent flows driven by Rayleigh-Taylor and Richtmyer-Meshkov instabilities. Phys. Fluids 13 (2), 538-544.

Zhou, Y., Robey, H. F. \& Buckingham, A. C. 2003 Onset of turbulence in accelerated high-Reynolds-number flow. Phys. Rev. E 67 (5). 